EGU2020-17904

https://doi.org/10.5194/egusphere-egu2020-17904

EGU General Assembly 2020

(c) Author(s) 2021. This work is distributed under

the Creative Commons Attribution 4.0 License.

\title{
The SO/PHI instrument on Solar Orbiter and its data products
}

Sami K. Solanki ${ }^{1}$, Johann Hirzberger ${ }^{1}$, Thomas Wiegelmann ${ }^{1}$, Achim Gandorfer ${ }^{1}$, Joachim Woch ${ }^{1}$, and José Carlos del Toro Iniesta ${ }^{2}$

${ }^{1}$ Max Planck Institute for Solar System Research, Göttingen, Germany (solanki@mps.mpg.de)

${ }^{2}$ Suchergebnisse Webergebnisse Instituto de Astrofísica de Andalucía, Granda, Spain

A central instrument of Solar Orbiter is the Polarimetric and Helioseismic Imager, SO/PHI. It is a vector magnetograph that also provides data for helioseismology. SO/PHI is composed of two telescopes, a full-disk telescope (FDT) and a high-resolution telescope (HRT). The HRT will observe at a resolution as high as $200 \mathrm{~km}$ on the solar surface, while the FDT will obtain the magnetic field and velocity of the full solar disc whenever it observes. SO/PHI will be the first solar spectropolarimeter to leave the Sun-Earth line, opening up some unique perspectives, such as the first detailed view of the solar poles. This will allow not just a more precise and exact mapping of the polar magnetic field than possible so far, but will also enable us to follow the dynamics of individual magnetic features at high latitudes and to determine solar surface and sub-surface flows right up to the poles. In addition to its standard data products (vector magnetograms, continuum images and maps of the line-of-sight velocity), SO/PHI will also provide higher-level data products. These will include synoptic charts, local magnetic field extrapolations starting from HRT data and global magnetic field extrapolations (from FDT data) with potential field sourcesurface (PFSS) models and possibly also non-potential models such as NLFFF (non-linear force-free fields), magnetostatics and MHD. The SO/PHI data products will usefully complement the data taken by other instruments on Solar Orbiter and on Solar Probe, as well as instruments on the ground or in Earth orbit. Combining with observations by Earth-based and near-Earth telescopes will enable new types of investigations, such as stereoscopic polarimetry and stereoscopic helioseismology. 